\title{
ATAXIA-TELANGIECTASIA PRESENTING WITH CRANIOSTENOSIS
}

BY

\author{
A. ROBINSON \\ From the Children's Department, St. Thomas's Hospital, London
}

(RECEIVED FOR PUBLICATION JUNE 15, 1962)

Ataxia-telangiectasia is a familial syndrome which gradually unfolds during early childhood. It is characterized by progressive cerebellar ataxia, symmetrical telangiectasia, frequent respiratory infections, delayed physical but normal mental development and quite often a dry skin with café-au-lait spots. The first published case by Louis-Bar (1941) was followed by fuller and welldocumented reports by Boder and Sedgwick (1957, 1958), Centerwall and Miller (1958) and Ford (1960), and leave no doubt that this is a clinical entity of which more than 80 cases are now known (E. Boder, personal communication, 1962).

A further case history of this condition is reported, which presented in an unusual way and in which investigations not previously reported have been carried out.

\section{Case History}

A boy, born in May 1957, first presented at the age of 2 years and 2 months with the story that 'he was not steady on his feet and backward in his speech'. The pregnancy and birth were normal and the immediate neonatal period was free from anoxia or jaundice. A squint which has persisted was noted at this time.

Progress was apparently normal during the first year. He sat alone at 6 months, stood at 9 months and walked unaided at 1 year. From this time his gait was said by his parents to be 'unsteady and trembling' with frequent falls. First recognizable words were noted at 19 months, but were never distinct.

When first seen he was obviously ataxic with a marked intention tremor of his hands and titubation of his head. He had a latent nystagmus, particularly on abduction, with a left internal strabismus. The fundi were normal. The tone and reflexes were considered normal and, although difficult to test, there was no gross abnormality of sensation. The skull was dolichocephalic and on palpation revealed marked heaping of the sagittal and coronal sutures. The impression was that in addition to his physical handicap he was also mentally backward. A clinical diagnosis of craniostenosis with congenital cerebellar ataxia was made. A skull radiograph confirmed the craniostenosis and surgery was performed on
July 22, 1959 (Mr. Harvey Jackson) for separation of the sagittal and coronal sutures.

His progress for the next year was considered to be good by both parents and doctors. His speech became more distinct but never clear, and his vocabulary developed normally. His gait became steadier, he did not fall so often, and he could manipulate his toys with considerable facility, although the tremor was always present. He even managed to feed himself with some difficulty.

During the first few months of 1961 a deterioration was noted. His gait became more awkward, he could not climb on to chairs and, most alarming to the parents, he could not raise himself from the lying to the sitting position. His speech during this period became more dysarthric.

On examination he was a timid but happy little boy, with perhaps some emotional lability and mental retardation. His face was held somewhat rigid with a slow smile and stereotype laughing and crying. His speech was moderately dysarthric, there was titubation of the head, intention tremor of the hands with an inconstant tremor at rest. The skull circumference was $20 \frac{1}{4}$ in. $(51.4 \mathrm{~cm}$.). The limbs were held semiflexed, and in the arms there was minimal wasting of the proximal muscles. All limbs were hypotonic, but especially in the legs full extension was limited with a cog-wheel type rigidity. Reflexes were normal. Although interpretation was difficult, it was considered that there was weakness in all muscle groups, particularly in the arms. He was barely able to feed himself and was occasionally doubly incontinent, whereas before he had been clean apart from wetting at night (Fig. 1).

It was then that the telangiectasia was first noted on the cheeks, the backs of the hands and the knees. Since this time there has been no further deterioration in the neurological signs, the telangiectasia has become more obvious and now includes the ears and bulbar conjunctivae. Throughout he has not been subject to excessive respiratory illnesses.

Investigations. As in all previous communications, these have been for the most part uninformative.

Routine blood tests, Wassermann and Kahn reaction, urine and urinary amino acids, serum proteins, phosphorus, creatinine and amine copper oxidase, C.S.F., 


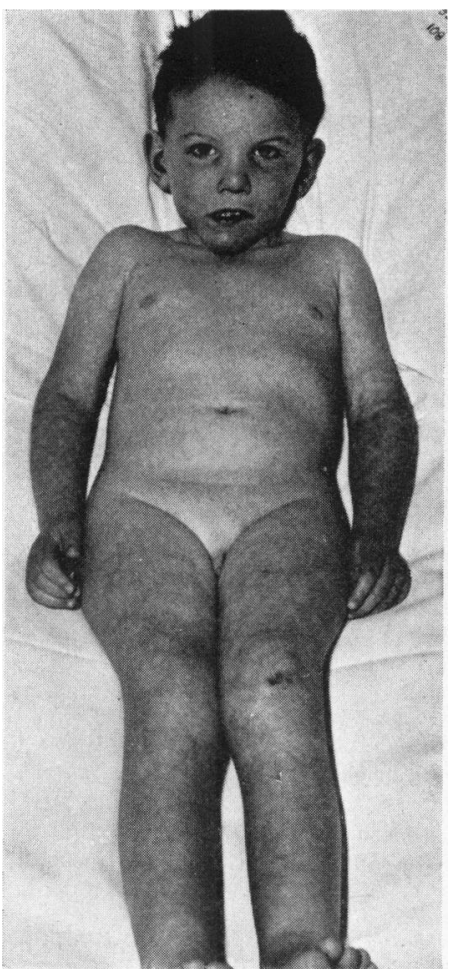

Fig. 1.-Rigid facies, flexed limbs and skin marbling of the patient.

E.E.G., E.C.G. and radiographs of the spine, chest and sinuses were all normal.

Radiograph of skull confirmed the craniostenosis (Fig. 2).

Skin biopsy confirmed the telangiectasia.

Muscle biopsy (left peroneus longus). 'Routine stained sections showed marked variations in muscle fibre calibre which was mainly focal in distribution, the majority of fibres being of small calibre. Some groups of fibres were extremely atrophic. There was a moderate increase in endomysial and perimysial fibrous tissue. Methylene blue stained sections showed variation in calibre of the distal nerve fibres with abnormal swelling of the axons. There was increased distal branching. These changes were considerably more marked in those nerves that supplied the atrophic muscle fibres. Motor endplates varied considerably in size and many showed degenerative changes, and sections stained for cholinesterase confirmed this. The appearances were those of a moderately severe neuropathy.' (Figs. 3 and 4.)

Electromyogram. Stimulation of the lateral popliteal nerve, with pick-up of the peroneus longus, showed considerable increased duration with ragged outline and a great deal of scatter.

Creatine phosphokinase: 1.6 units (normal).

Cholinesterase time: $14 \mathrm{~min}$. (normal 7-18 min.).

Blood pyruvic acid: $1.4 \mathrm{mg} . / 100 \mathrm{ml}$. (not fasting).

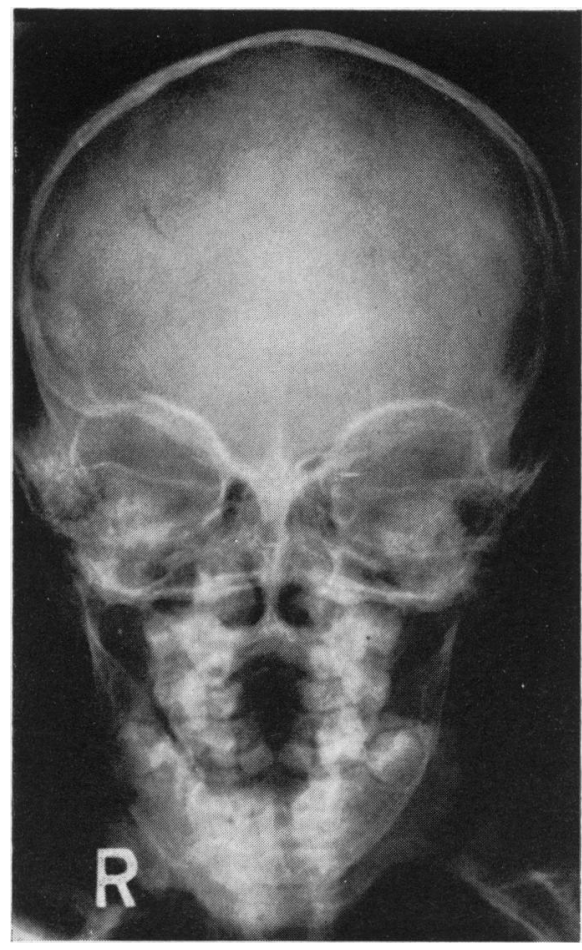

Fig. 2.-Skull radiograph in 1959, showing absence of sagittal suture.

Serum magnesium: $1.7 \mathrm{mEq} / \mathrm{l}$. (normal $1 \cdot 5-1 \cdot 8$ $\mathrm{mEq} / 1$.$) .$

Serum aldolase: normal.

Serum mercury: less than $0.5 \mu \mathrm{g} . / 100 \mathrm{ml}$.

I.Q. June 12, 1961. Attempts were made to assess intelligence on the Terman and Merrill test. He was considered to be very close to average, although not functioning at that level because of his severe physical handicap.

\section{Discussion}

Awareness of this admittedly rare syndrome is important if the patient is to escape unnecessary and unrewarding diagnostic procedures such as air encephalography, vertebral angiography and craniotomy, which might otherwise be considered necessary.

The key to the diagnosis is the telangiectasia. In all cases (except the original one of Louis-Bar) this follows the ataxia, often by several years. It is during this period that the diagnostic difficulties arise. Earlier recognition is possible only if the more subtle beginnings are looked for in any case of cerebellar ataxia presenting in infancy or early childhood. This would also encourage more 


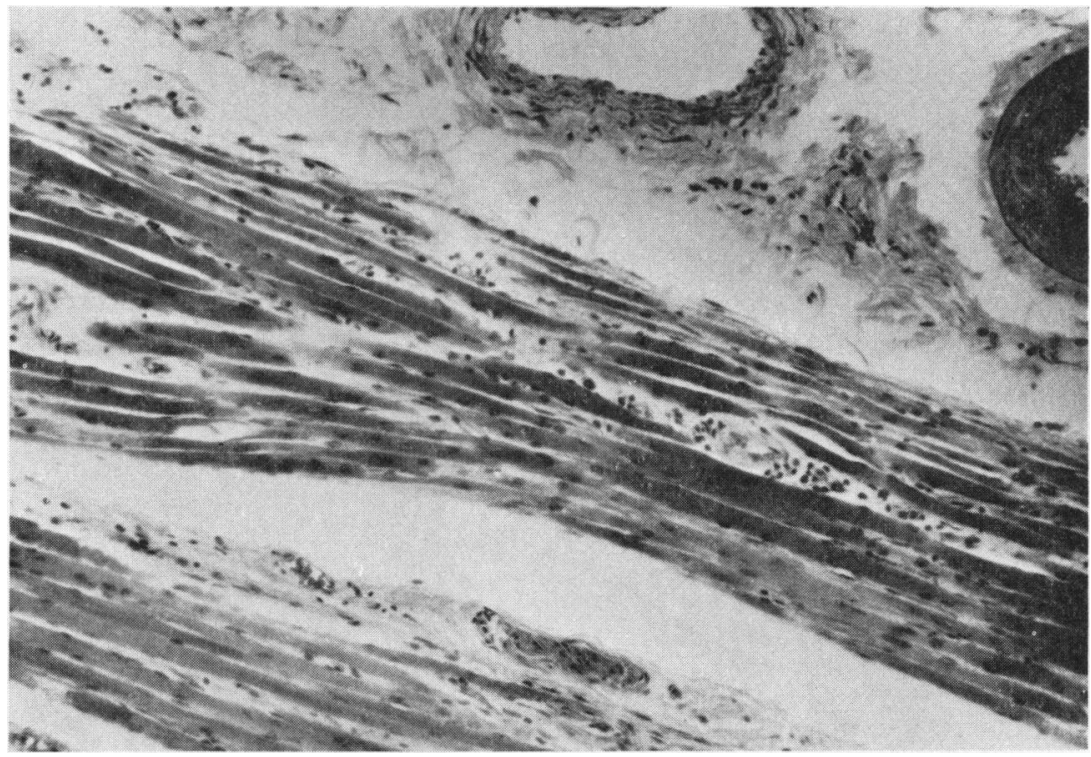

FIG. 3.--Muscle biopsy, showing marked variation in fibre calibre. (H. and E. $\times$ 120.)

vigorous treatment for the respiratory infections to which these children are prone and would avoid errors in labelling them superficially as mentally backward. On the other hand, investigations endeavouring to throw light on the aetiology are still necessary. For these reasons we have presented this case.

Ataxia is not a feature of the neurological complications of craniostenosis and indeed when first seen was not thought to be a factor in the symp-

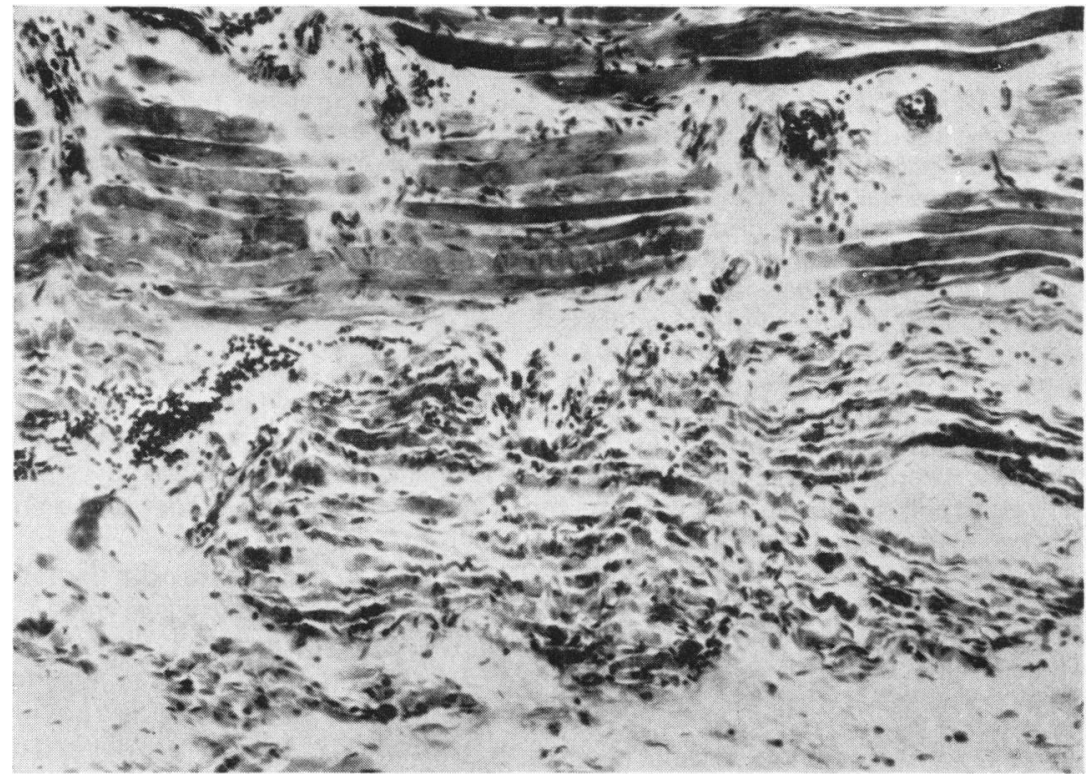

Fig. 4.-Muscle biopsy, showing focal necrosis.

(H. and E. $\times$ 120.) 
tomatology. The improvement following surgery may well have been a reflection of the increase in motor skills usual at this age and thus tending to mask the gradual increase in the ataxia.

Boder and Sedgwick investigated the possibility of an abnormality in heavy metal metabolism, as in Wilson's disease. They found serum iron and copper and urinary lead to be normal. It was thought worth while to extend this to cover mercury and magnesium, as poisoning with these metals produces ataxia, and both were normal. A muscle biopsy was done because of the significant and rapid onset of muscle weakness and minimal wasting with early contracture noted during the 'relapse' phase. These features have not been reported previously and microscopy indicates that there is a neuromuscular component to this syndrome. The serum aldolase which is raised in some muscular dystrophies was also normal. The possibility that the central and peripheral nerve lesions might be related to an abnormality of cholinesterase and related enzymes was investigated with negative results.

The exact aetiology remains obscure, but one is not unmindful of similar changes that develop in carcinomatous neuromyopathy in adults, which are presumably metabolic in origin (Brain and Henson, 1958).

\section{Summary}

A case of ataxia-telangiectasia is reported. An unusual feature was associated craniostenosis. Abnormal findings of a muscle biopsy are reported. Serum estimations of mercury and magnesium were found to be normal as was the serum aldolase, creatine phosphokinase and cholinesterase time.

I should like to thank Dr. B. D. R. Wilson for permission to publish this case, and Dr. $\mathrm{H}$. Barrie for his constant encouragement. Thanks are also due to Dr. V. MacDermot for interpretation of the muscle biopsy, and Mr. J. S. Fenton of the Anatomy Department for the photomicrographs.

\section{REFERENCES}

Boder, E. and Sedgwick, R. P. (1957). Ataxia-telangiectasia: a familial syndrome of progressive cerebellar ataxia, oculocutaneous telangiectasia and frequent pulmonary infection. Univ. South Calif. med. Bull. 9, 15 .

, (1958). Ataxia-telangiectasia. A familial syndrome and frequent pulmonary infection. Pediatrics, 21, 526.

Brain, R. and Henson, R. A. (1958). Neurological syndromes associated with carcinoma: The carcinomatous neuromyopathies. Lancet, 2, 971 .

Centerwall, W. R. and Miller, M. M. (1958). Ataxia, telangiectasia, and sinopulmonary infections. A syndrome of slowly progressive deterioration in childhood. A.M.A.J. Dis. Child., 95, 385 .

Ford, F. R. (1960). Diseases of the Nervous Sistem in Infancy, Childhood and Adolescence, 4th ed., p. 944. Thomas, Springfield, Illinois.

Louis-Bar (1941). Sur un syndrome progressif comprenant des télangiéctasies capillaires cutanées et conjonctivales symétriques, neurol. (Basel), 4, 32. 